\title{
Effects of unilateral or bilateral superior ovarian nerve section in infantile rats on follicular growth
}

\author{
C Morán, L Morales, U Quiróz and R Domínguez \\ Unidad de Investigación en Biología de la Reproducción, Laboratorio de Fisiología de la Reproducción, Facultad de Estudios Superiores Zaragoza, \\ UNAM AP 9-020, CP 15000, México, DF México \\ (Requests for offprints should be addressed to C Morán, Facultad de Estudios Superiores Zaragoza, UNAM. AP 9-020, CP15000, México, DF México)
}

\begin{abstract}
We report the effects that sectioning the superior ovarian nerve of infantile female rats has on their follicular development at different ages before puberty. Compared with the control group, sham-operated animals showed a significant decrease in the number of measured follicles in right and left ovaries, although no difference in the follicular atresia ratio was observed. Animals with a sectioned left superior ovarian nerve (SON), killed 12 days after surgery had a significant increase in the number of follicles in the ovaries. Most of the follicles were atretic. Sectioning the right SON induced contrasting effects in the ovaries of animals killed 4 and 16 days after surgery. Rats with a denervated (right) ovary showed a decrease in the number of follicles and a greater number of atretic follicles compared with the control group, whereas the
\end{abstract}

innervated (left) ovary showed an increase in measured follicles compared with the control group.

Bilateral sectioning had no apparent effect on the total number of follicles measured, although an increased number of atretic follicles in both ovaries was observed. Animals with a unilateral section of the SON, killed 8 and 12 days after surgery, showed a decrease in serum concentrations of estradiol. In turn, animals killed 16 days after surgery showed a significant increase in estradiol and a decrease in the progesterone serum concentration.

These results suggest that sympathetic innervation of the ovary via the SON has a stimulatory role in the regulation and differentiation of follicular growth.

Journal of Endocrinology (2000) 166, 205-211

\section{Introduction}

Ovaries receive sympathetic innervation (norepinephrine and vasoactive intestinal peptide (VIP)) through the superior ovarian nerve and the plexus nerve (norepinephrine and substance P) (Aguado \& Ojeda 1984a). Both norepinephrine and peptides act via adenylate-cyclasecoupled receptors, which are present in the ovary at the beginning of folliculogenesis (Ben-Jonathan et al. 1984, Advis et al. 1989) and before the newly formed follicles acquire follicle stimulating hormone (FSH) receptors. VIP has been shown to induce cAMP formation and to stimulate aromatase activity in feto-neonatal ovaries that are unresponsive to gonadotropins (George \& Ojeda 1987).

Different studies have reported the participation of the sympathetic innervation in the regulation of ovarian steroidogenesis (Kawakami et al. 1981), ovulation (Chávez et al. 1991, Morales et al. 1993) and follicular growth (Grob 1974, Curry et al. 1984). According to Hirshfield (1992), the first follicles to start growing are those assembled near the ovarian hilum, which, in the ovaries of the rat, is the first region to be innervated during its feto-neonatal life (Malamed et al. 1992). In addition, evidence of the close relationship of catecholaminergic nerve terminals to the theca layer of the ovary has been reported (Burden 1978, Greenwald \& Roy 1994).

According to Lara et al. (1990), sympathetic chemical denervation induced by the injection of guanethidine to rats, starting on the 7th day after birth, resulted in an increase of the number of antral follicles and a decrease in the number of preantral follicles. In turn, Riboni et al. (1998), reported that injection of guanethidine to newborn female guinea pigs resulted in an increase of the mean number of follicles measured in the ovaries of denervated animals and an increase in the follicular atresia. Morales et al. (1993) found that unilateral sectioning of the superior ovarian nerve of 16-day-old rats resulted in a significant decrease in the number of ova shed by the denervated ovary and a compensatory ovulation by the innervated one. Sequential injection of gonadotropins did not restore ovulation by the denervated ovary (Morales et al. 1998).

Treatment of new-born guanethidine-denervated rats with pregnant mare's serum gonadotropin-human chorionic gonadotropin at days 18 or 21, induced ovulation, but this response was not observed when guanethidine was injected to 15-day-old rats (Flores et al. 1998) - results that 
suggest that innervation has different roles during different developmental stages.

In this study, we analysed the effects of unilateral and bilateral sectioning of the superior ovarian nerve on the regulation of follicular development and hormonal secretion before puberty, and its relation with the reduction of ovarian response to gonadotropins and induction of ovulation.

\section{Materials and Methods}

One hundred and seventy-two 16-day-old rats of the CIIZ-V strain from our own breeding stock were used. Animals were maintained under controlled lighting conditions (lights on from 0500 to $1900 \mathrm{~h}$ ) and had free access to rat chow pellets and tap water. Pups were weaned on the 21st postnatal day and housed in group cages (five females and one male per cage). Groups of eight or nine animals were randomly assigned to the following experimental groups: (1) sham-operated animals (SO); (2) sectioning of the left superior ovarian nerve (left SON); (3) sectioning of the right superior ovarian nerve (right $\mathrm{SON}$ ); (4) bilateral sectioning of the superior ovarian nerve (bilateral SON); (5) untouched animals (control group)

Sectioning of the superior ovarian nerve and shamoperation procedures were performed between 0900 and $1000 \mathrm{~h}$. Animals were anesthetised with ether and one or both ovaries were exposed through a lateral incision. With the aid of fine forceps, the ovarian ligament was sectioned at approximately $1 \mathrm{~cm}$ from the ovary. Sham surgery involved laparotomy.

As first vaginal opening and first vaginal estrus occur at 34 days after birth and establish the onset of puberty in female rats, experimental treatment animals were killed by decapitation 4, 8, 12 and 16 days after surgery. Control group animals were killed at $20,24,28$ or 32 days of age. During decapitation, the blood from the trunk was collected, allowed to clot and centrifuged at 3000 r.p.m. The serum was stored at $-20{ }^{\circ} \mathrm{C}$.

Serum concentration of estradiol $(\mathrm{pg} / \mathrm{ml})$ and progesterone $(\mathrm{ng} / \mathrm{ml})$ were measured by radioimmunoassay, using kits purchased from Diagnostic Products (Los Angeles, CA, USA). The intra- and interassay coefficients of variation were $2 \cdot 1 \%$ and $5 \cdot 6 \%$ for progesterone and $6 \cdot 0 \%$ and $7 \cdot 1 \%$ for estradiol, respectively.

At autopsy, ovaries and uterus were dissected and weighed in a precision balance. The ovaries were fixed in Bouin's solution, embedded in paraffin wax, serially sectioned at $10 \mu \mathrm{m}$ thickness and stained with hematoxylin-eosin.

All sections from three randomly chosen ovaries of each group were examined microscopically. Follicle diameter was measured from follicles in which the nucleus and nucleolus of the oocyte were present. Measurements were taken from basement membrane to basement membrane, using a calibrated ocular micrometer. Mean diameter was obtained by adding the maximum diameter plus the diameter taken at right angles.

Follicles were classified as healthy or atretic. A follicle was considered to be undergoing atresia when one of the following characteristics was observed: presence of the piknosis in the granulosa cell, granulosa cell present in the follicular fluid, or hypertrophy of the theca cells.

\section{Statistical analysis}

Data on the weight of ovaries and uterus (in mg), estradiol and progesterone concentrations and for the differences in mean follicular diameter $(\mu \mathrm{m})$ were analysed by multivariate analysis of variance (MANOVA), followed by either Tukey's test or Student's $t$-test. The difference in the number of follicles was analysed by Kruskal-Wallis test, followed by Mann-Whitney U-test. Follicular distribution was analysed by $\chi^{2}$ test. A probability of less than $5 \%$ was considered significant.

\section{Results}

Figures 1, 2 and 3 present data on the number of follicles and the percent of atresia measured in the ovaries of untouched control, sham-operated and unilaterally or bilaterally SON-sectioned animals killed 4, 8, 12 or 16 days after treatment.

A decrease in the number of follicles was measured in the ovaries of animals that underwent sham operation (left and right ovary) and were killed 4 and 8 days later. This decrease was observed in the left ovary when the animals were killed 12 days after laparotomy, but was not observed in the right ovary. Differences in the number of follicles in untouched control and sham-operated animals killed 16 days after laparotomy were not observed. The distributions of normal and atretic follicles of left and right ovaries were not modified by sham procedures (Fig. 1A,B).

In comparison with sham-operated animals in the left SON group, a significant increase in atretic follicles in the denervated ovary was observed 4 days after section (Fig. 1C). Animals killed 12 days after sectioning of the left SON showed an increase in the number of follicles measured in both ovaries (Fig. 1A, B), most of them being atretic (Fig. 1C, D). When the animals were killed 16 days after surgery, a decrease in the number of follicles measured in the right ovary was observed (Fig. 1B).

Animals killed 4 and 16 days after sectioning of the right SON exhibited a decrease in the number of follicles measured in the right ovary, but those killed 12 days after right $\mathrm{SON}$ showed an increase in the number of follicles in the innervated (left) ovary (Fig. 2A, B). A significant increase was observed in the number of atretic follicles in the right and left ovaries of animals killed on day 12 after surgery (Fig. 2C, D). The denervated ovary of the animals killed 4 days after treatment presented an increase in atresia (Fig. 2D). 

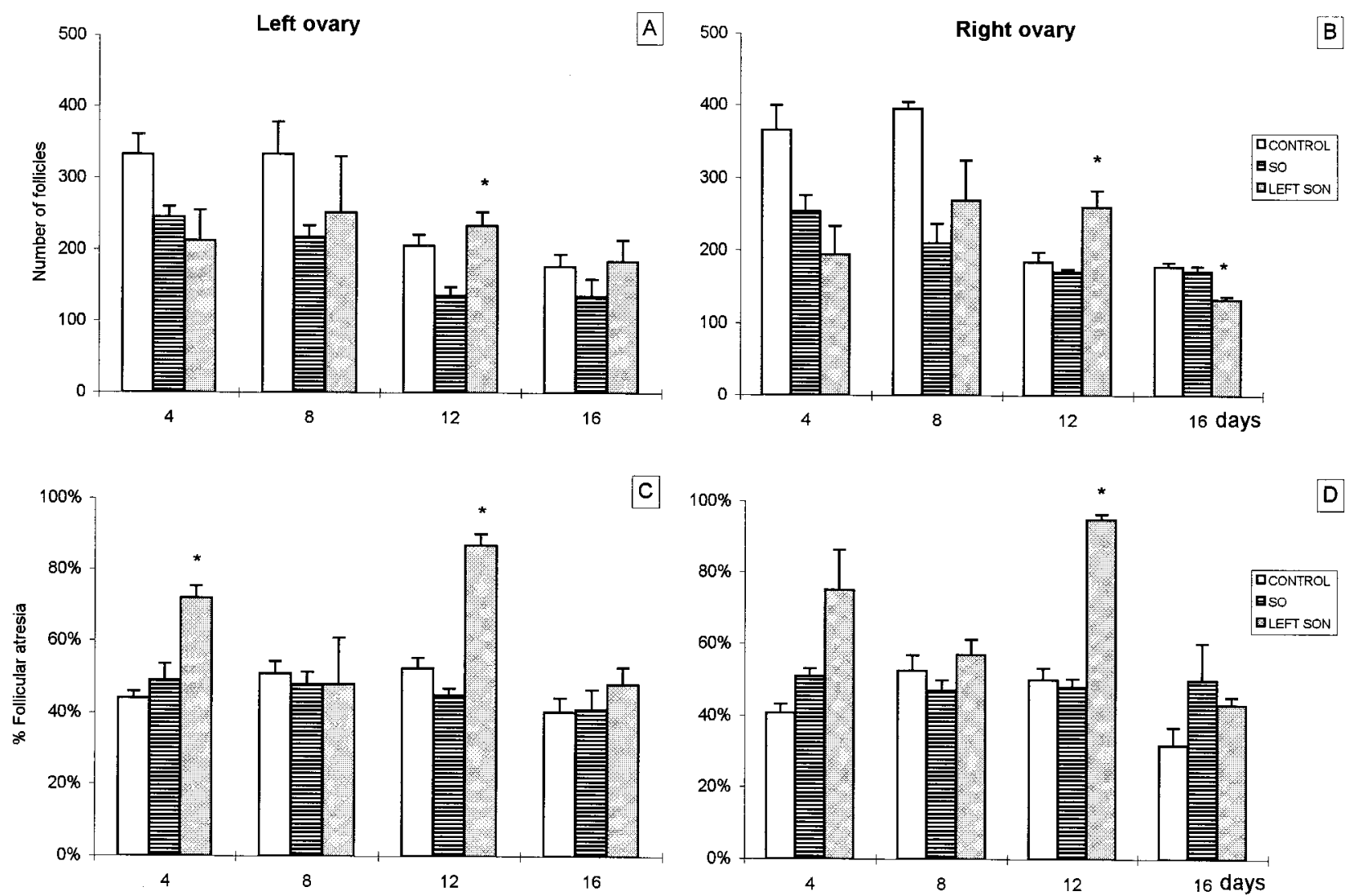

Figure 1 Number of follicles (A, B) and percentage follicular atresia (C, D) in control and sham-operated animals (SO) and those in which left section of the superior ovarian nerve (left SON) was performed 16 days after birth, killed 4, 8, 12 or 16 days after laparotomy. Values are means \pm S.E.M. ${ }^{*} P<0 \cdot 05$ compared with SO group (MANOVA).

Bilateral denervation had no apparent effect on the number of follicles measured in either ovary, when the animals were killed 4,8 or 12 days after surgery. A significant decrease was observed in the number of follicles measured in the right ovary 16 days after treatment (Fig. 3A, B). Animals killed 4, 8 and 12 days after denervation showed an increase in the number of atretic follicles in both ovaries (Fig. 3C, D).

Animals with a sectioned left SON, killed 12 days after denervation, had decreased serum estradiol concentrations, whereas both left SON- and right SON-sectioned animals killed 16 days after surgery showed a significant increase in serum estradiol. No significant changes in uterine weight were observed.

Bilateral denervation did not affect serum estradiol concentrations, but these animals showed a significant decrease in the weight of the uterus (Table 1).

Animals with a sectioned left SON killed 12 days after surgery showed a significant increase in serum progesterone concentrations. Unilateral and bilateral sectioned animals killed 16 days after denervation exhibited a significant decrease in serum progesterone concentrations, compared with those in sham-operated animals (Table 1).
In contrast, compared with the control group, shamoperated animals killed 16 days after laparotomy had significantly greater ovarian weights. Right SONsectioned animals killed 4 days after denervation had significantly greater ovarian weights than the shamoperated group. These differences were almost absent in animals killed 8 or 12 days after surgery. However, animals with a bilateral section killed 16 days after surgery (prepubertal period) showed a significant decrease in the weight of their right ovary (Table 2 ).

\section{Discussion}

The results suggest that sympathetic innervation of the ovary via the $\mathrm{SON}$ has a modulatory role in the regulation of follicular differentiation and growth. Right and left ovaries respond in distinct ways to different experimental situations (Chávez et al. 1987, Domínguez 1990, D’Albora et al. 1992, Gerendai et al. 1995, Cassina \& Domínguez 1996, Gerendai \& Halasz 1997), and these differences have been explained by differences in the ovarian innervation (Domínguez et al. 1989). The differences in follicular growth and differentiation induced by sectioning of the 

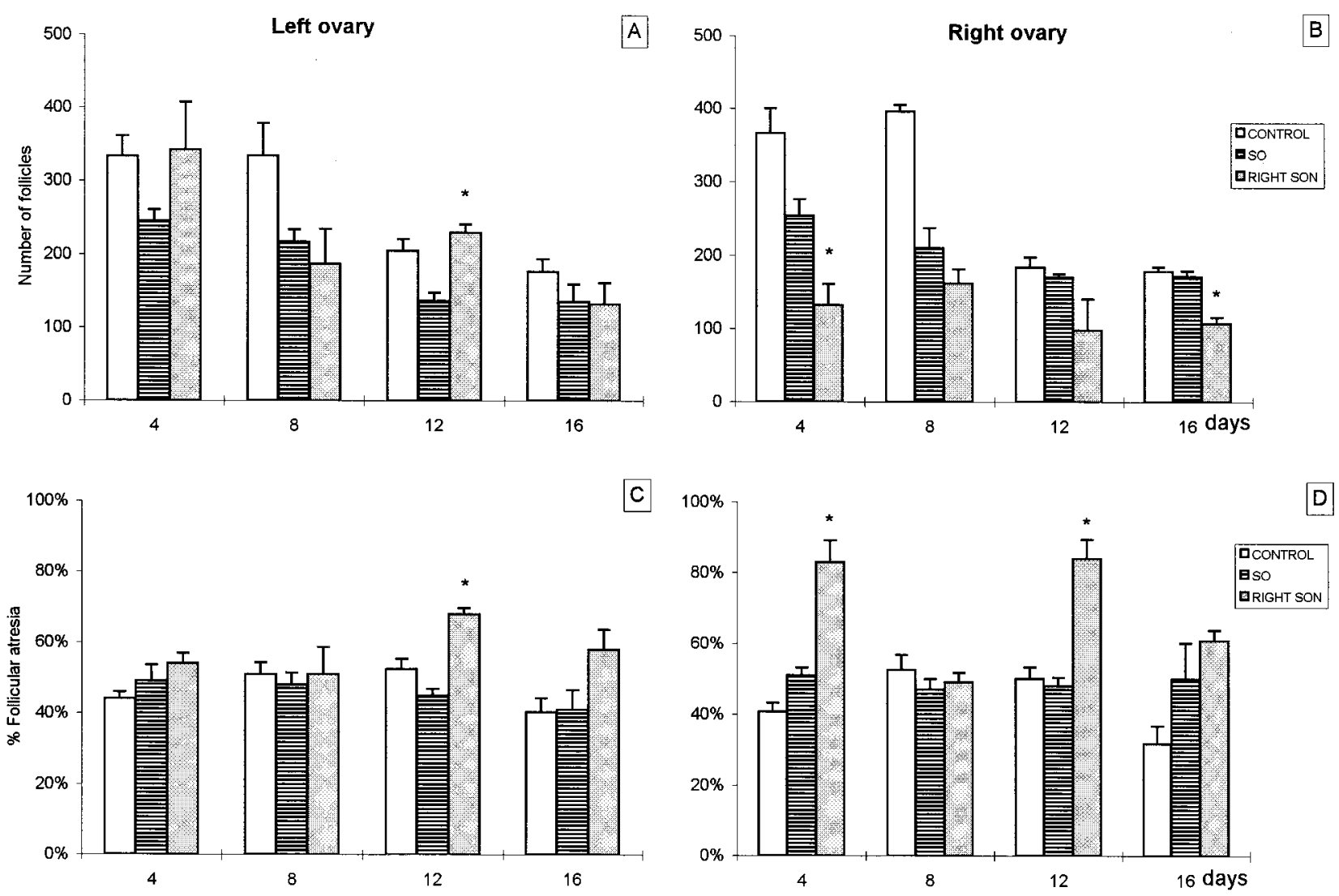

Figure 2 Number of follicles (A, B) and percentage follicular atresia (C, D) in control and sham-operated animals (SO) and those in which right section of the superior ovarian nerve (right SON) was performed 16 days after birth, killed 4, 8, 12 or 16 days after surgery. Values are means \pm S.E.M. ${ }^{*} P<0 \cdot 05$ compared with $\mathrm{SO}$ group (MANOVA).

right or left SON in innervated and denervated ovaries support such an interpretation.

Animals that underwent right SON and were killed 12 days after surgery, showed a decrease in the number of follicles measured in the denervated ovary, an increase in the number of follicles in the (innervated) left ovary, and a high degree of atresia in the right ovary. These results may explain the low number of ova shed by the denervated ovary, and the compensatory ovulation of the innervated ovary observed (Morales et al. 1993). However, animals with a sectioned left SON, did not conform to this explanation, as both ovaries responded in a similar way. Furthermore, Morales et al. (1993) reported that animals with a sectioned left SON exhibited low numbers of ova shed by the left ovary and compensatory ovulation by the right ovary.

Ferruz et al. (1991) suggested that the release of norepinephrine by ovarian nerve endings may be regulated by gonadotropins. Because animals with unilateral section of the SON showed a decrease in ovulation from the denervated ovary, while significantly increasing ovulation from the innervated one, the existence of a possible increase in sympathetic tone in the innervated ovary may have resulted in the increase in the number of ova shed. Previously, we have shown that unilateral section of the SON results in a significant difference in norepinephrine concentration between the innervated and denervated ovaries (Chávez et al. 1994). Another plausible explanation for the different response between right and left ovaries, is the presence of 'neural communication' between ovaries, where the signal, arising from the left ovary goes to the right ovary, as previously proposed (Chávez et al. 1994). In this context, Advis et al. (1989) have shown that, in hypophysectomised immature rats, the concentration of VIP in the left ovary increased after a lesion to the right, left or bilateral side of the preoptic-hypothalamic anterior area, whereas concentrations of this peptide in the right ovary remained unchanged. According to Aguado \& Ojeda (1984a), bilateral sectioning of the SON produced a twofold increase in the number of ovarian adrenergic receptors - an increase that might compensate for the loss of neural input to the ovary. Such an interpretation could explain the lack of follicular growth observed in animals with bilateral section of the SON in the present study. 

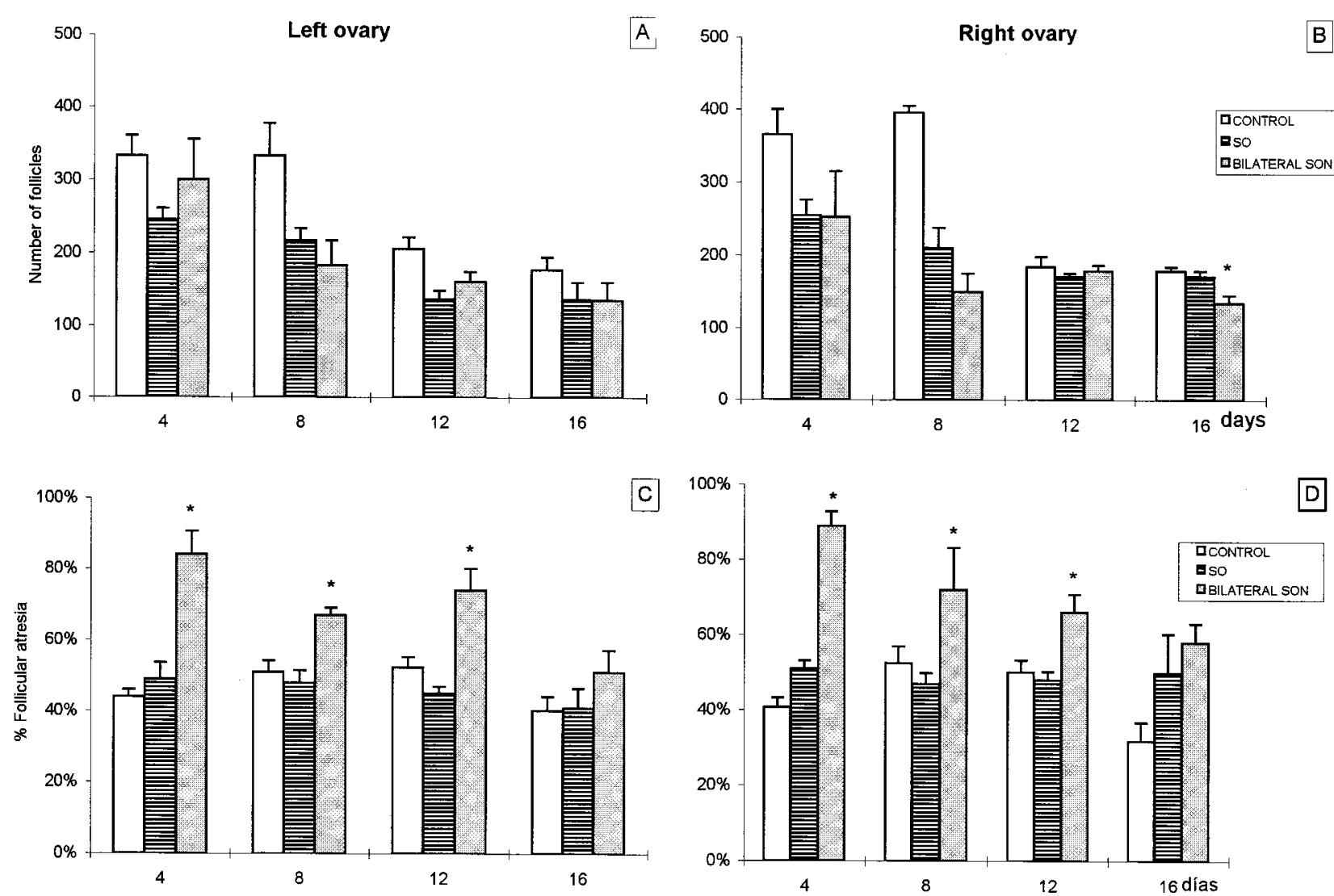

Figure 3 Number of follicles (A, B) and percentage follicular atresia (C, D) in control and sham-operated animals (SO) and those in which bilateral section of the superior ovarian nerve (bilateral SON) was performed 16 days after birth, killed 4, 8, 12 or 16 days after surgery. Values are means \pm S.E.M. ${ }^{*} P<0 \cdot 05$ compared with $S O$ group (MANOVA).

Table 1 Mean \pm S.E.M. serum concentrations of estradiol and progesterone in control rats, sham-operated rats (SO) and animals with section of the left (Left SON), right (Right SON) or both (Bilateral SON) superior ovarian nerve performed at 16 days of age, killed $4,8,12$ or 16 days after surgery

\begin{tabular}{|c|c|c|c|c|c|}
\hline & \multirow[b]{2}{*}{$n$} & \multicolumn{4}{|c|}{ Time after surgery (days) } \\
\hline & & 4 & 8 & 12 & 16 \\
\hline \multicolumn{6}{|l|}{ Oestradiol (pg/ml) } \\
\hline $\mathrm{SO}$ & 9 & $35 \cdot 0 \pm 11 \cdot 8$ & $12 \cdot 4 \pm 1 \cdot 8^{*}$ & $14 \cdot 8 \pm 1 \cdot 8^{*}$ & $11 \cdot 3 \pm 1 \cdot 7$ \\
\hline Left SON & 8 & $43 \cdot 2 \pm 12 \cdot 4$ & $17 \cdot 9 \pm 3 \cdot 2$ & $7 \cdot 8 \pm 1 \cdot 4^{* \dagger}$ & $30 \cdot 8 \pm 6 \cdot 1^{\dagger}$ \\
\hline Right SON & 9 & $32 \cdot 8 \pm 5 \cdot 3$ & $15 \cdot 8 \pm 5 \cdot 1$ & $20 \cdot 6 \pm 4 \cdot 4$ & $43 \cdot 0 \pm 8 \cdot 5^{\star \dagger}$ \\
\hline Control & 8 & $6 \cdot 6 \pm 1 \cdot 4$ & $2 \cdot 3 \pm 0 \cdot 3$ & $18 \cdot 6 \pm 7 \cdot 0$ & $12 \cdot 8 \pm 1 \cdot 8$ \\
\hline $\mathrm{SO}$ & 9 & $4 \cdot 4 \pm 0 \cdot 8$ & $1 \cdot 2 \pm 0 \cdot 2^{*}$ & $2 \cdot 6 \pm 0 \cdot 5^{*}$ & $10 \cdot 4 \pm 2 \cdot 9$ \\
\hline Left SON & 8 & $8 \cdot 3 \pm 1 \cdot 9$ & $1 \cdot 9 \pm 0 \cdot 4$ & $5 \cdot 4 \pm 0 \cdot 6^{\dagger}$ & $3 \cdot 9 \pm 0 \cdot 8^{\dagger *}$ \\
\hline Right SON & 9 & $5 \cdot 3 \pm 1 \cdot 5$ & $2 \cdot 4 \pm 0 \cdot 4^{\dagger}$ & $3 \cdot 6 \pm 0 \cdot 9$ & $3 \cdot 3 \pm 1 \cdot 3^{\dagger *}$ \\
\hline Bilateral SON & 9 & $2 \cdot 6 \pm 0 \cdot 6^{*}$ & - & $6 \cdot 0 \pm 2 \cdot 6$ & $2 \cdot 5 \pm 1 \cdot 7^{\dagger *}$ \\
\hline
\end{tabular}

$P<0 \cdot 05$ compared with: * control group, ${ }^{\dagger} \mathrm{SO}$ (MANOVA, followed by Tukey's test).

Another possible explanation of the changes observed in animals with unilateral section of the superior ovarian nerve is that modifications occurred in the blood flow to the ovaries, produced by the section. Such an explanation seems implausible, however, as Aguado \& Ojeda (1984b) reported that bilateral sectioning of the superior ovarian nerve did not affect ovarian blood flow. 
Table 2 Mean \pm S.E.M. weights of the left ovary (LO), right ovary (RO) and uterus in control rats, sham-operated rats (SO) and animals with section of the left (left SON), right (Right SON) or both (Bilateral SON) superior ovarian nerve performed at 16 days of age, killed 4, 8,12 or 16 days after surgery

\begin{tabular}{|c|c|c|c|c|c|c|}
\hline & & \multicolumn{5}{|c|}{ Time after surgery (days) } \\
\hline \multicolumn{7}{|c|}{ Ovarian weight (mg) } \\
\hline Control & RO & 8 & $10 \cdot 4 \pm 1 \cdot 2$ & $13 \cdot 8 \pm 0 \cdot 7$ & $10 \cdot 3 \pm 0 \cdot 6$ & $9 \cdot 5 \pm 0 \cdot 3$ \\
\hline \multirow[t]{2}{*}{ SO } & LO & 9 & $8 \cdot 7 \pm 0 \cdot 6$ & $12 \cdot 1 \pm 0 \cdot 5$ & $13 \cdot 6 \pm 1 \cdot 6$ & $15 \cdot 9 \pm 1 \cdot 6^{*}$ \\
\hline & RO & 9 & $8 \cdot 8 \pm 0 \cdot 6$ & $13 \cdot 7 \pm 1 \cdot 2$ & $13 \cdot 5 \pm 3 \cdot 2$ & $15 \cdot 0 \pm 1 \cdot 0^{*}$ \\
\hline \multirow[t]{2}{*}{ Right SON } & LO & 9 & $12 \cdot 0 \pm 0 \cdot 8^{\dagger}$ & $15 \cdot 4 \pm 0 \cdot 6^{\dagger}$ & $15 \cdot 4 \pm 1 \cdot 4$ & $18 \cdot 1 \pm 1 \cdot 0^{*}$ \\
\hline & RO & 9 & $12 \cdot 0 \pm 0 \cdot 9^{\dagger}$ & $14 \cdot 4 \pm 2 \cdot 3$ & $9 \cdot 9 \pm 1 \cdot 3$ & $11 \cdot 7 \pm 1 \cdot 0$ \\
\hline \multirow[t]{2}{*}{ Bilateral SON } & LO & 9 & $12 \cdot 4 \pm 1 \cdot 0^{\dagger}$ & $11 \cdot 0 \pm 0 \cdot 5$ & $13 \cdot 6 \pm 0 \cdot 9$ & $11 \cdot 4 \pm 1 \cdot 1$ \\
\hline & RO & 9 & $11 \cdot 5 \pm 1 \cdot 3$ & $12 \cdot 1 \pm 2 \cdot 6$ & $12 \cdot 2 \pm 0 \cdot 6$ & $11 \cdot 1 \pm 0 \cdot 8^{\dagger}$ \\
\hline \multicolumn{7}{|c|}{ Uterine weight (mg) } \\
\hline Control & & 8 & $32 \cdot 9 \pm 1 \cdot 1$ & $37 \cdot 5 \pm 3 \cdot 2$ & $38 \cdot 7 \pm 4 \cdot 2$ & $85 \cdot 1 \pm 5 \cdot 4$ \\
\hline
\end{tabular}

P<0.05 compared with: * ${ }^{*}$ control group, ${ }^{\dagger} \mathrm{SO}$ (MANOVA, followed by Tukey's test).

Differences observed between sham-operated and untouched control animals could be explained by longlasting stress induced by anesthesia and skin section. We have previously shown that, in adult rats, the same treatment resulted in a diminution of serum concentrations of FSH and luteinizing hormone that lasted several days (Flores et al. 1990).

In vitro studies have suggested that VIP increases the number of FSH receptors (Mayerhofer et al. 1997), and that sectioning the SON and ovarian plexus results in a significant decrease in ovarian concentrations of VIP (Advis et al. 1989). Therefore, the increased number of atretic follicles in animals with unilateral or bilateral sectioning of the SON may be explained by a decrease in FSH receptors, as a result of denervation.

Sympathetic innervation stimulates ovarian steroidogenesis, whereas the bilateral sectioning of the SON in the morning of the proestrus day resulted in an immediate diminution of plasma concentrations of estradiol and progesterone, with normal values thereafter (Aguado \& Ojeda 1984b, Erskine \& Weaver 1988). Thus the observed differences in serum concentrations of estradiol and progesterone in animals with unilateral or bilateral section of the SON could be explained by a re-arrangement of the neural factors that regulate ovarian steroidogenesis.

\section{Acknowledgements}

This work was supported by CONACyT grants $29006 \mathrm{~N}$ and DGAPA IN203799.

\section{References}

Advis JP, Ahmed CE \& Ojeda SR 1989 Direct hypothalamic control of vasoactive intestinal peptide (VIP) levels in the developing rat ovary. Brain Research Bulletin 22 605-610.

Aguado LI \& Ojeda SR 1984a Prepubertal ovarian function is finely regulated by direct adrenergic influences. Role of noradrenergic innervation. Endocrinology 114 1845-1853.

Aguado LI \& Ojeda SR $1984 b$ Ovarian adrenergic nerves play a role in maintaining preovulatory steroid secretion. Endocrinology 114 1944-1946.

Ben-Jonathan N, Arbogast LA, Rhoades TA \& Bahr J 1984 Norepinephrine in the rat ovary: ontogeny and de novo synthesis. Endocrinology 115 1426-1431.

Burden HW 1978 Ovarian innervation. In The Vertebrate Ovary: Comparative Biology, pp 615-638. Ed RE Jones. New York: Plenum Press.

Cassina P \& Domí nguez R 1996 The effects of reserpine administration on compensatory ovulation and compensatory ovarian hypertrophy in prepubertal hemiovariectomized rats depend on the in situ ovary. Medical Science Research 24 597-599.

Chávez R, Cruz ME \& Domínguez R 1987 Differences in the ovulation rate of the right or left ovary in unilaterally ovariectomized rats: effect of ipsi and contralateral vagus nerves on the remaining ovary. Journal of Endocrinology 113 397-401.

Chávez R, Carrizosa L \& Domínguez R 1991 Effects of superior ovarian nerve section on spontaneous and induced ovulation in adult rats. Medical Science Research 19 41-42.

Chávez R, Morales L, González ME \& Domínguez R 1994 Ovarian norepinephrine content in prepubertal rats with superior ovarian nerve section: temporal studies. Medical Science Research 22 789-790.

Curry TE, Lawrence IE \& Burden HW 1984 Ovarian sympathectomy in the guinea pig II. Effects on follicular development during the prepubertal period and following exogenous gonadotropin stimulation. Cell and Tissue Research 236 593-596.

D’Albora H, Cassina MP, Barreiro JP, Sapiro R \& Domínguez R 1992 Differences in follicular growth and ovulation ability in the 
autografted right and left ovary of hemiovariectomised prepubertal rats. Medical Science Research 20 755-757.

Domínguez R, Cruz ME \& Chávez R 1989 Differences in the ovulatory ability between the right and left ovary are related to ovarian innervation. In Growth Factors and the Ovary, pp 321-325. Ed AN Hirshfield. New York: Plenum Press.

Domínguez R 1990 Differential ovulatory responses of the right and left ovaries of the adult rat to unilateral lesion and anaesthesia of the cervico-vaginal plexus. Journal of Endocrinology 124 43-45.

Erskine MS \& Weaver ChE 1988 The role of ovarian sympathetic innervation in the control of estrous responsiveness in the rat. Hormones and Behavior 22 1-11.

Ferruz J, Barria A, Galleguillos X \& Lara HE 1991 Release of norepinephrine from the rat ovary: local modulation by gonadotropins. Biology of Reproduction 45 592-597.

Flores A, Morales L, Ulloa-Aguirre A \& Domínguez R 1990 Acute changes in serum levels of luteinising hormone and follicle stimulating hormone, ovulation and follicular growth induced by stress, unilateral ovariectomy or mechanical stimulation of the ovarian pedicle at different stages of the oestrous cycle of the rat. Medical Science Research 18 819-821.

Flores A, Loyo R \& Domínguez R 1998 Peripheral sympathetic denervation induced by guanethidine in newborn rats results in the advancement of ovulation but does not modify oestrogen positive feedback effects. Medical Science Research 26 259-261.

George FW \& Ojeda SR 1987 Vasoactive intestinal peptide enhances aromatase activity in the neonatal rat ovary before development of primary follicles or responsiveness to follicle-stimulating hormone. PNAS 84 5803-5807.

Gerendai I \& Halasz B 1997 Neuroendocrine asymmetry. Frontiers in Neuroendocrinology 18 354-381.

Gerendai I, Csaba Zs, Vokó Z \& Csernus V 1995 Involvement of a direct neural mechanism in the control of gonadal functions. Journal of Steroid Biochemistry and Molecular Biology 53 299-305.

Greenwald GS \& Roy SK 1994 Follicular development and its control. In The Physiology of Reproduction edn 2, pp 629-724. Eds E Knobil \& JD Neill. New York: Raven Press.
Grob HS 1974 Follicle growth in denervated ovaries of hypophysectomized mice. Federation Proceedings 33213.

Hirshfield AN 1992 Heterogeneity of cell populations that contribute to the formation of primordial follicles in rats. Biology of Reproduction 47 466-472.

Kawakami M, Hayashi R, Kubo K, Nagasse M \& Uekura T 1981 Involvement of ovarian innervation in steroid secretion. Endocrinology 109 136-145.

Lara HE, McDonald JK, Ahmed CE \& Ojeda SR 1990 Guanethidinemediated destruction of ovarian sympathetic nerves disrupts ovarian development and function in rats. Endocrinology 127 2199-2209.

Malamed S, Gibney JA \& Ojeda SR 1992 Ovarian innervation develops before initiation of folliculogenesis in the rat. Cell and Tissue Research 270 87-93.

Mayerhofer A, Dissen GA, Costa ME \& Ojeda SR 1997 A role for neurotransmitters in early follicular development: induction of functional follicle-stimulating hormone receptors in newly formed follicles of the rat ovary. Endocrinology 138 3320-3329.

Morales L, Chávez R \& Domínguez R 1993 Participation of superior ovarian nerve in the regulation of ovulation in the prepubertal rat: differential effects of unilateral and bilateral section of the nerve. Medical Science Research 21 15-17.

Morales L, Chávez R, Ayala ME \& Domínguez R 1998 Effects of the unilateral or bilateral superior ovarian nerve section in prepubertal rats, on the ovulatory response to gonadotrophins administration. Journal of Endocrinology 158 213-219.

Riboni L, Escamilla C, Chavira R \& Domínguez R 1998 Effects of peripheral sympathetic denervation induced by guanethidine administration on the mechanisms regulating puberty in the female guinea pig. Journal of Endocrinology 156 91-98.

Received 2 August 1999

Revised manuscript received 27 January 2000

Accepted 21 March 2000 